\title{
Human Immunodeficiency Virus Type 1 Brazilian Subtype B Variant Showed an Increasing Avidity of the Anti-V3 Antibodies over Time Compared to the Subtype B US/European Strain in São Paulo, Brazil
}

\author{
Jorge Casseb $^{+}$, Patricia Montanheiro, Shirley Komninakis, Adriana Brito, \\ Alberto JS Duarte
}

\begin{abstract}
Laboratório de Alergia e Imunologia Clínica e Experimental, Faculdade de Medicina, Universidade de São Paulo,
\end{abstract} R. Dr. Enéas de Carvalho 500, Prédio II, 05403-000 São Paulo, SP, Brasil

The Brazilian variant of human immunodeficiency virus type 1 (HIV-1) subtype B, (serotype B"-GWGR), has a tryptophan replacing the proline in position 328 the HIV-1 envelope. A longer median time period from infection to acquired immunodeficiency syndrome (AIDS) for serotype B $(B$ "'-GWGR) infected subjects compared to the B-GPGR US/European strain was reported. In a cohort study, in São Paulo city, 10 B"-GWGR patients had a statistically significant increased avidity of the anti-V3 antibodies, from $79 \% \pm 33 \%$ to $85 \% \pm 75 \%$, versus from $48 \% \pm 59 \%$ to $32 \% \pm 17 \%$ for the $10 \mathrm{~B}-G P G R$ subjects $(p=0.02)$. The TCD4+cells showed a mean increase of +0.45 cells $/$ month for the B-GPGR subjects and for B"-GWGR the slope was +1.24 cells/month $(p=0.06)$, for 62 and 55 months of follow up, respectively. RNA plasma viral load decreased from $3.98 \pm 1.75$ to $2.16 \pm 1.54 \log _{10}$ in the B"-GWGR group while B-GPGR patients showed one $\log _{10}$ reduction in viral load from $4.09 \pm 0.38$ to $3.17 \pm 1.47 \log _{10}$ over time $(p=0.23)$, with a decreasing slope of $-0.0042 \pm \log _{10}$, /month and $-0.0080 \pm \log _{10} /$ month, for B-GPGR and $B$ "-GWGR patients, respectively $(p=0.53)$. Neither group presented any AIDS defining events during the study, according to Center for Diseases Control criteria. Although the sample size is small, these results may indicate that differences in the pathogenicity of the 2 HIV-1 B serotypes which co-circulate in Brazil may be correlated to the avidity of anti-V3 antibodies.

Key words: human immunodeficiency virus type 1 (HIV-1) - Brazilian HIV-1 variant - V3 serotyping - antibody avidity São Paulo - Brazil

The Brazilian variant of human immunodeficiency virus type 1 (HIV-1) subtype B, named serotype B"-GWGR, has a unique signature in the tip of the V3 loop, with tryptophan replacing proline in position 328 in the HIV-1 envelope (Louwagie et al. 1994). Molecular typing and V3 serology approaches showed that this variant accounts for almost half of the subtype B infection in Brazil (Louwagie et al. 1994, Casseb et al. 1998). Recently, Santoro-Lopes et al. (2000) reported a longer median time to acquired immunodeficiency syndrome (AIDS) among serotype B (B"-GWGR) infected subjects compared to the B-GPGR US/European strain. A cohort study in São Paulo, we also found a 2-fold decreased risk of AIDS development among asymptomatic, untreated patients with B"GWGR serology versus B-GPGR serology (Casseb et al. 2002). The possible difference in pathogenesis has still to

Financial support: Fapesp 97/11502-2, 98/00261-7, 99/111881 , and CNPq 301224/2000-5

${ }^{+}$Corresponding author. Fax: +55-11-3081.7190. E-mail: j31@ hotmail.com

Received 3 September 2003

Accepted 9 January 2004 be explained. To address this point, we have used a previously described V3 serologic assay to identify the Brazilian variant and to assess the avidity of V3 antibodies (Hendry el al. 1996, Casseb et al. 1998). Biotinylated peptides based on the V3 loop consensus sequence from subtype B (Consensus B: N T R K S I H I G P G R A F Y), and 1 synthetic peptide based on the consensus sequence of the Brazilian variant subtype B strain (Strain BR1: N T R K S I H I G W G R A) were captured onto avidin-coated 96 well plates. Serial 4-fold dilutions of test sera were added to duplicate plates, 1 of which was washed 5 times with a hiperosmolar $(8 \mathrm{M})$ urea solution, and the other plate with 5 washings in PBS. Peroxidase-labeled anti-human $\operatorname{IgG}$ was added, incubated for $1 \mathrm{~h}$, and the reaction was developed with the substrate hydrogen peroxide and the tetrametilbenzidine chromogen. The plates were read at $450 \mathrm{~nm}$ and the end-point titers were interpolated from the linear portion of the titration curve yielding a mean absorbance of 0.5 optical density units. The high avidity anti-V3 antibodies index (HAAV3 index) was calculated using the dilution end-point in the $8 \mathrm{M}$ ureawashed plate divided by the dilution end-point in the PBSwashed plate. Antibodies with HAAV3 index greater than $50 \%$ were considered to possess higher affinity. One serum sample from a patient who has not been under going 
any treatment in the last 5 years was used as a control for the assays. T CD4+ cell counts were performed by flow cytometry with commercial monoclonal antibodies (Beckman Coulter, Miami, FL, US) and RNA plasma viral load was quantified using NASBA kits (Organon Teknika, Durham, NC, US). From a total of 157 HIV-1-infected subjects, we evaluated serial plasma samples collected from 20 asymptomatic HIV-infected individuals (10 individuals with B-GPGR and 10 with B"-GWGR motif) with similar T CD4+ cell count at baseline, with sera from the 3 samples collected at different time points available in our specimen repository. These samples were chosen randomly, the only criteria was that only patients with sera in the 3 time points [1995-1997, when combined anti-retroviral therapy (ART) was used on $1 \mathrm{~B}$ "-GWGR, 5 patients were undergoing 2 drug regimens, 3 under unknown treatment, 4 with out any ART and the remaining patients taking AZT only; after 24 months of ART for all but 1 patient still not under; and last evaluation available time point after start of ART] from our cohort were included. This cohort has been followed up in the Ambulatory of the Secondary Immunodeficiency Clinic, Department of Dermatology of the Hospital das Clínicas, School of Medicine of the São Paulo University. More details regarding this cohort have been published elsewhere (Fonseca et al. 1999, Brigido et al. 2001). The statistical analysis was done using a nonparametric test (Mann-Whitney's test) to compare the groups, and the results presented in mean and standard deviation values, obtained with samples collected at the last available point (year 2000).

The Table shows the demographic data and laboratory results. The B"-GWGR patients had a statistically significant, increased HAAV3 index, from $79 \% \pm 33 \%$ to $85 \% \pm 75 \%$ (mean $\pm \mathrm{SD}$ ), versus $48 \% \pm 59 \%$ and $32 \% \pm$ $17 \%$ for the B-GPGR group, $(\mathrm{p}=0.02)$. The T CD $4+$ cells increased +0.45 cells/month for the B-GPGR subjects and for B"-GWGR the slope was +1.24 cells/month $(p=0.06)$, for $62 \pm 40$ and $55 \pm 16$ months of follow up, respectively. RNA plasma viral load decreased from $3.98 \pm 1.75$ to 2.16 $\pm 1.54 \log _{10}$ in the B"-GWGR group while B-GPGR patients showed only one $\log _{10}$ reduction in viral load (from $4.09 \pm 0.38$ to $\left.3.17 \pm 1.47 \log _{10}\right)$ over time ( $\left.\mathrm{p}=0.23\right)$, with a decreasing slope of $-0.0042 \log _{10}, /$ month and -0.008 $\log _{10} /$ month, for B-GPGR and B"-GWGR patients, respectively ( $p=0.53$ ) (Figure). Neither group presented any AIDS defining events during the study, according to the CDC criteria (CDC 1987). The untreated control subject had no change in T CD4+ cell count, plasma viremia or HAAV3 index over time. Neither time of use nor numbers of anti-retrovirals employed, such as triple therapy including one protease inhibitor, did differ significantly between the 2 serotype groups (data not shown).

The B"-GWGR patients showed a $2-\log _{10}$ decrease in their viral load, while the B-GPGR patients showed only 1 $\log _{10}$ decrease. Thus, it may indicate that differences in the pathogenicity of the 2 HIV-1 B serotypes which cocirculate in Brazil may be correlated to the avidity of antiV3 antibodies (Broström et al. 1995). One possible explanation is that the conformational changes caused by the replacement of the proline amino acid by a tryptophan may lead to a decreased infectivity. However, biological

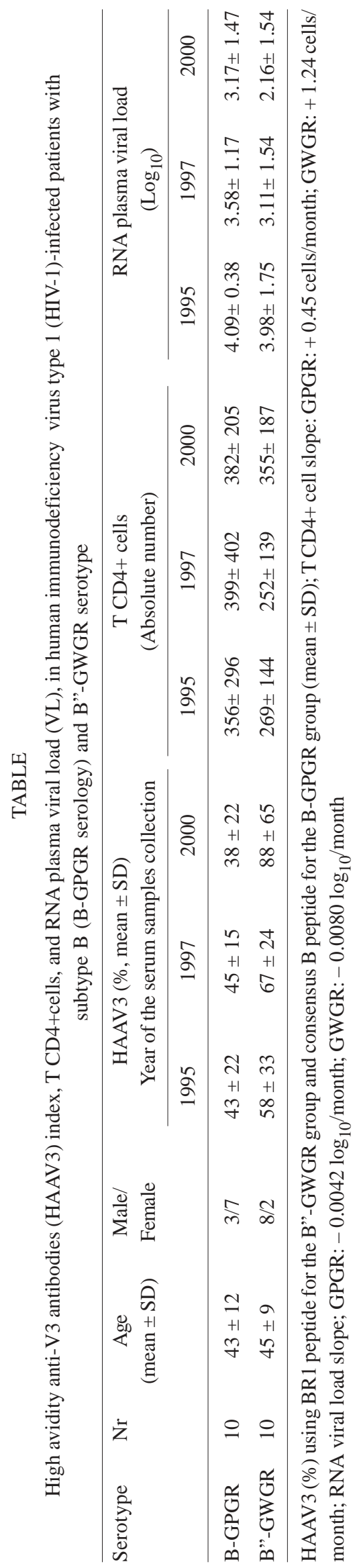




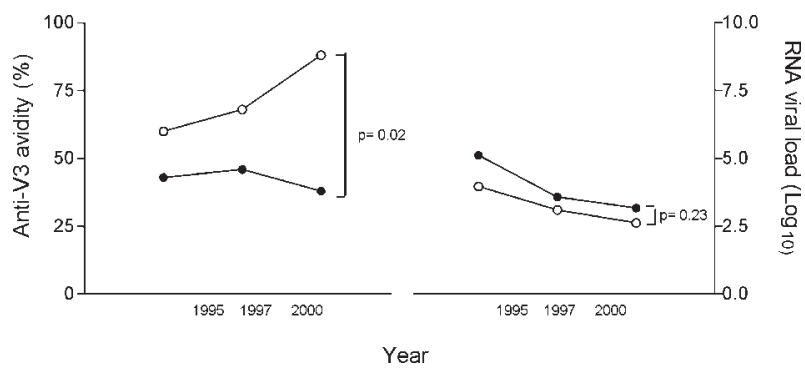

High avidity anti-V3 antibodies index and RNA viral load in BGPGR and B"-GWGR patients. Results expressed in mean, obtained of sera samples from 10 patients of each group (B-GPGR vs B"GWGR motif). GPGR sera reactivity to consensus B peptide (GPGR motif) and GWGR sera reactivity to BR1 peptide (GWGR motif)

and co-receptor usage studies are necessary to address these findings.

More recently, with the highly active antiretroviral therapy (HAART) use, neutralizing antibodies were noted to be a marker for immune reconstitution. In fact, 1 study showed that prior to starting therapy, the HAART success group (VL below detection limit) had significantly higher levels of HIV-1 neutralizing antibodies than the HAART failure group (VL above detection limit). Also, anti-V3 binding titers to several V3 peptides were higher in the HAART success group. After 1 year of treatment, neutralizing antibody levels decreased and the response became more specific in the HAART success group. Just the opposite occurred in the HAART failure group. It has been shown that about $50 \%$ of patients who receive HAART actually have complete viral suppression, and it is possible that HIV-specific humoral immune response contributes to the success or failure of HAART (Morris et al. 2001).

Despite the high diversity of V3 loop region in Brazilian samples, especially in the external tetrade, the key position for antibody reactivity is driven by the position occupied by proline or tryptophan (data not published). We also considered the possibility of male/female ratio as a potential confounder, as well as the different time entries in the cohort. To minimize these confounders, a slope curve was done over time to homogenize the groups.

Finally, our results indicated a higher HAAV3 index among patients with reactivity to B"-GWGR peptides than did B-GPGR patients. However, there is not reasonable explanation, but this finding provides additional information on the pathogenesis of subtype B in Brazil and further studies should be done using larger sample size.

\section{REFERENCES}

Brigido L, Rodrigues R, Menezes PR, Oliveira D, Rossetti M, Menezes P, Duarte AJS 2001. Impact of the adherence to antiretroviral therapy (ART) in HIV-1-Iinfected users of an university public service in São Paulo City, Brazil. AIDS Patient Care and STDs 15: 587-593.

Broström C, Sonnenberg A, Sallberg M 1995. Human immunodeficiency virus (HIV) type 1 -infected patients with no disease progression display high-avidity production to autologous V3 sequences. J Infect Dis 171: 509-511.

Casseb J, Hong MA, Gonsalez C, Brigido LF, Duarte AJ, Hendry RM 1998. Two variants of HIV-1 B serotype are transmitted heterosexually in São Paulo, Brazil. Braz J Med Biol Res 31: 1243-1246.

Casseb J, Komninakis S, Abadalla L, Brigido LFM, Rodrigues R, Araújo F, Veiga ARV, Almeida A, Hendry RM, Duarte AJS 2002. HIV disease progression: is the Brazilian variant subtype B' (GWGR motif) less pathogenic than US/European subtype B (GPGR)? Inter J Infect Dis 6: 64-69.

CDC-Centers for Disease Control and Prevention 1987. Revision of the surveillance case definition for acquired immunodeficiency syndrome. MMWR 36(Suppl.1S): 1S-15S.

Fonseca LA, Reingold AL, Casseb J, Brigido LFM, Duarte AJS 1999. AIDS incidence and survival in a hospital-based cohort of asymptomatic HIV asymptomatic patients in São Paulo, Brazil. Int J Epidemiol 28: 1156-1160.

Hendry RM, Hanson CV, Bongertz V, Morgado M, Duarte A, Casseb J, Brigido L, Sabino E, Diaz R, Galvão-Castro B 1996. Immunoreactivity of Brazilian HIV isolates with different V3 motifs. Mem Inst Oswaldo Cruz 91: 347-348.

Louwagie J, Delwart EL, Mullins JI, McCutchan FE, Eddy G, Burke DS 1994. Genetic analysis of HIV-1 isolates from Brazil reveals the presence of two distinct genotypes. AIDS Res Hum Retroviruses 10: 561-567.

Morris MK, Katzenstein DA, Israelski D, Zolopa A, Hendry RM, Hanson CV 2001. Characterization of the HIV-1 specific humoral immune response during highly active antiretroviral therapy (HAART). J Acquir Immune Defic Syndr 15: 405-415.

Santoro-Lopes G, Harrison LH, Tavares MD, Xexeo A, Dos Santos AC, Schechter M 2000. V3-binding serotypes and HIV disease progression in Brazil: is B different from B'. AIDS Res Hum Retroviruses 16: 953-958. 